Check for updates

Cite this: RSC Adv., 2018, 8, 6719

\title{
An alginate film-based degradable triboelectric nanogenerator $\dagger$
}

\author{
Yaokun Pang,,$^{\mathrm{ab}}$ Fengben Xi, $\stackrel{t}{\mathrm{t}}^{\mathrm{ab}}$ Jianjun Luo, ${ }^{\mathrm{ab}}$ Guoxu Liu, ${ }^{\mathrm{ab}}$ Tong Guo ${ }^{\mathrm{abc}}$ \\ and Chi Zhang (D)*ab
}

Alginate, as a natural linear polysaccharide derived from brown sea algae, has the advantage of low toxicity, good biocompatibility, and biodegradability, which has aroused wide interests in recent years. In this study, a degradable triboelectric generator based on an alginate film is presented. The calcium alginate film, which is prepared by a simple freeze-drying method and a crosslinking reaction, has a form of porous structures that are beneficial for triboelectric power generation. The fabricated TENG has a stable output performance with a maximum voltage, current, and power of $33 \mathrm{~V}, 150 \mathrm{nA}$, and $9.5 \mu \mathrm{W}$, respectively. The performances of the TENG were investigated at different thicknesses of the calcium alginate film and various concentrations of the sodium alginate solution, as well as the degradability of the film with different thicknesses and temperatures. In addition, the TENG was designed for harvesting water wave energy in a low-frequency range from 1 to $4 \mathrm{~Hz}$. This study is promising to provide new insights to develop degradable and ecofriendly TENG based on ocean plants and expand the application range in blue energy.

Received 13th December 2017 Accepted 31st January 2018

DOI: 10.1039/c7ra13294h

rsc.li/rsc-advances electrodes can be corroded and are harmless to the environment. ${ }^{27,28}$ The most widely used polymers, such as PTFE, Kapton, PDMS, and PET, in the TENG cannot be completely degraded and are liable to release hazardous chemicals. Therefore, use of degradable materials, especially, which are renewable and easily prepared, is very important for the application of TENG in the ocean.

As an important ocean biological resource, alginate is a family of copolymers produced by brown sea algae, possessing a linear chain of $\alpha$-L-guluronic acid and $\beta$-D-mannuronic acid. ${ }^{29}$ Due to the advantages of non-toxicity, biocompatibility, biodegradability, rich source, and low-cost, alginate has been widely used in the textile industry, wound dressings, food processing, artificial skin, and drug carriers. ${ }^{30-34}$ The most interesting property of alginates is their ability to react with calcium ions to form calcium alginate, which exhibits the so-called egg-box model with good mechanical strength. Calcium alginate is an environmentally friendly and renewable material that can be degraded in the ocean. The degraded material can be absorbed by the ocean plants or animals and is harmless to the environment, which may have significant implications in TENG.

Herein, we have developed a degradable triboelectric nanogenerator based on an alginate film. The calcium alginate film is extracted and fabricated from ocean plants. Using the simple freeze-drying method, the calcium alginate film has been obtained that has the porous structures that are beneficial for triboelectric power generation. The fabricated TENG has a stable output performance with a maximum voltage, current, and power of $33 \mathrm{~V}, 150 \mathrm{nA}$, and $9.5 \mu \mathrm{W}$, respectively. The performances of the TENG have been investigated at different

\footnotetext{
${ }^{a}$ CAS Center for Excellence in Nanoscience, Beijing Institute of Nanoenergy and Nanosystems, Chinese Academy of Sciences, National Center for Nanoscience and Technology (NCNST), Beijing 100083, China. E-mail: czhang@binn.cas.cn

${ }^{b}$ School of Nanoscience and Technology, University of Chinese Academy of Sciences, Beijing 100049, P. R. China

${ }^{\circ}$ Department of Physics and Laboratory of Material Physics, Zhengzhou University, Zhengzhou 450052, China

$\dagger$ Electronic supplementary information (ESI) available. See DOI: 10.1039/c7ra13294h

\$ These authors contributed equally to this work.
} 
thicknesses of the calcium alginate film and various concentrations of the sodium alginate solution. Furthermore, the calcium alginate film can be degraded in the ocean, and the influences of thickness and temperature on the degradability are investigated. The alginate film-based degradable triboelectric nanogenerator originates from the ocean initially and is absorbed by the ocean finally; this can be a self-cycle and environmentally friendly energy ecosystem. Finally, a sealed degradable triboelectric nanogenerator was designed and fabricated to harvest water wave energy. The vibrational frequency and unit number-dependent output characteristics were revealed. This study not only provides new insights to develop the degradable and eco-friendly TENG based on the ocean plants, but also expands the application range in blue energy.

\section{Results and discussion}

A schematic representing the fabrication of the degradable alginate film is shown in Fig. 1a. Sodium alginate is extracted from the ocean plants and has a linear chain of $\alpha$-L-guluronic acid and $\beta$-D-mannuronic acid; the chemical structural formula is shown in the insets. In the first step, the chemically puregrade sodium alginate powders are dissolved in deionized water while being stirred constantly at room temperature until the powders have fully dissolved. Then, the sodium alginate solution is transferred to a culture dish. It is worth noting that the culture dish surface is hydrophobic, and the contact angle with the sodium alginate solution is $98.6^{\circ}$, as shown in Fig. S1a, $\uparrow$ which is not suitable to form a thinner sodium alginate film and also affects the output performance of the TENG. After the UV-ozone treatment, as shown in Fig. S1b, $\uparrow$ the culture dish surface becomes hydrophilic with the contact angle of $34.5^{\circ}$, and it is easy to form the thinner sodium alginate film. The solution is pre-frozen at $-5{ }^{\circ} \mathrm{C}$ for at least $24 \mathrm{~h}$ to ensure the elimination of the entrained air. Subsequently, the frozen sodium alginate is lyophilized in a freezing dryer at $-55{ }^{\circ} \mathrm{C}$ for $24 \mathrm{~h}$. The fabricated sodium alginate film has poor strength and flexibility, which is not appropriate as a friction material in TENG. Thus, the fabricated sodium alginate film is directly steeped in a $5 \%(\mathrm{w} / \mathrm{w})$ calcium chloride solution to cross-link for $10 \mathrm{~min}$, and then, the film is naturally dried at room temperature. As the chemical structural formula shows, calcium ions can exchange with sodium ions, and the chelate can exchange with the oxygen atoms, which make the alginate chains bound firmly together and synergy stronger. Owing to the chain interaction, calcium alginate forms a three-dimensional network structure, which is called the egg-box model. Fig. $1 \mathrm{~b}$

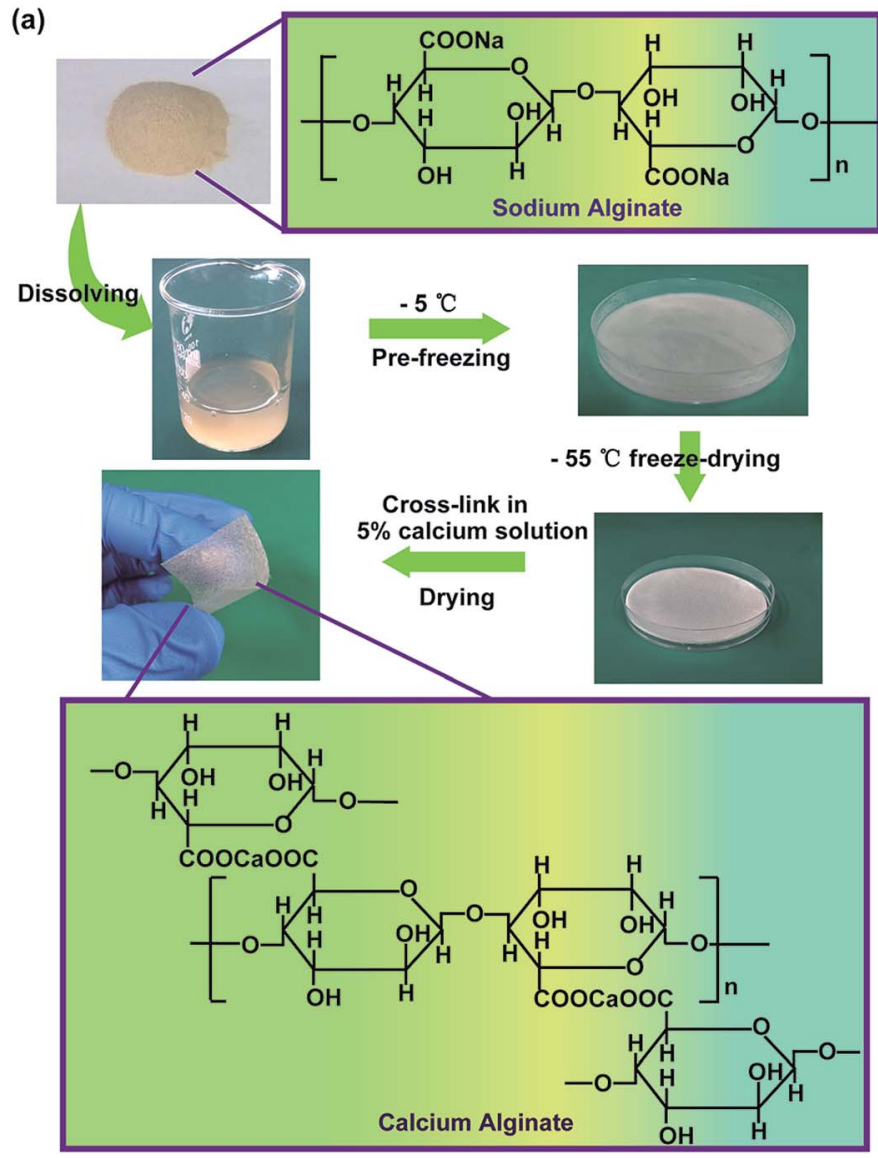

(b)

(c)

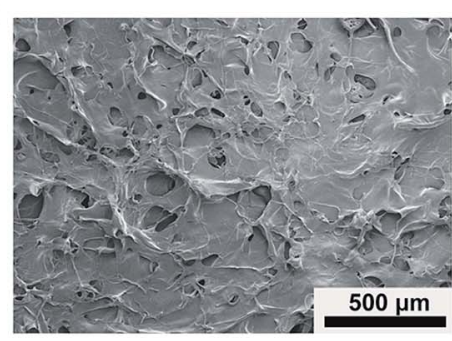

(d)
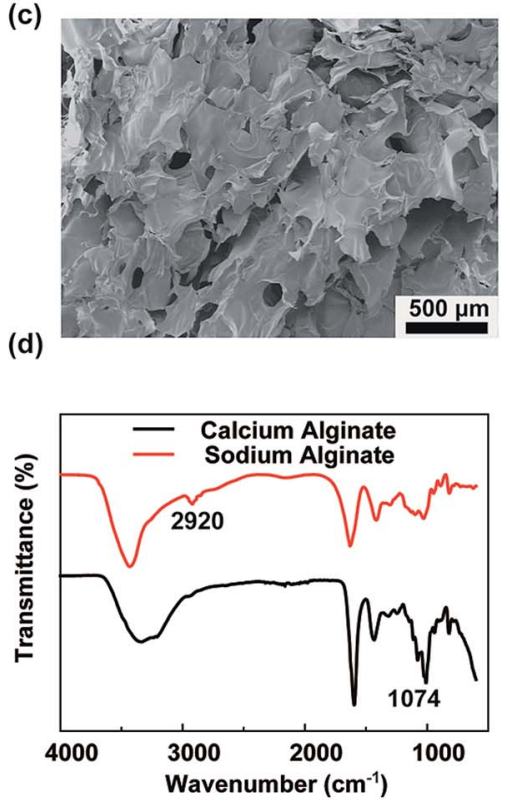

Fig. 1 Synthesis and characterization of the calcium alginate film. (a) Fabrication process for the calcium alginate film and the chemical structural formula in the insets. (b) Surface SEM image of the calcium alginate film. (c) Sectional SEM image of the calcium alginate film. (d) FTIR spectra of calcium alginate and sodium alginate. 
and c show the surface and sectional scanning electron microscopy (SEM) images of the calcium alginate film. It can be seen clearly that the surface is rough, has a high effective surface area, and can improve the triboelectric charge density. Using the freeze-drying method, the porous structures have been formed in the calcium alginate film after the removal of deionized water, which can effectively reduce the film weight and increase the film tensile strength. Fig. 1d shows the FTIR spectra of calcium alginate and sodium alginate. It can be seen that sodium alginate has an absorption band at $2920 \mathrm{~cm}^{-1}$ that is assigned to the stretching vibration of $\mathrm{C}-\mathrm{H}$ in the sixmembered ring. Owing to the cross-linking with calcium ion and formation of the egg-box model, the stretching vibration of $\mathrm{C}-\mathrm{H}$ in the six-membered ring of the calcium alginate molecule is limited, and the absorption band at $2920 \mathrm{~cm}^{-1}$ cannot be observed in the spectrum. In the sodium alginate molecule, the stretching vibration absorption band of - $\mathrm{COO}-$ and $\mathrm{C}-\mathrm{O}$ is very weak. However, in the calcium alginate molecule, the $\mathrm{C}-\mathrm{O}-\mathrm{Ca}-$ $\mathrm{O}-\mathrm{CO}-$ structure makes the $\mathrm{C}-\mathrm{O}$ stretching vibration absorption increase and has an obvious absorption band at $1074 \mathrm{~cm}^{-1}$.

(a)
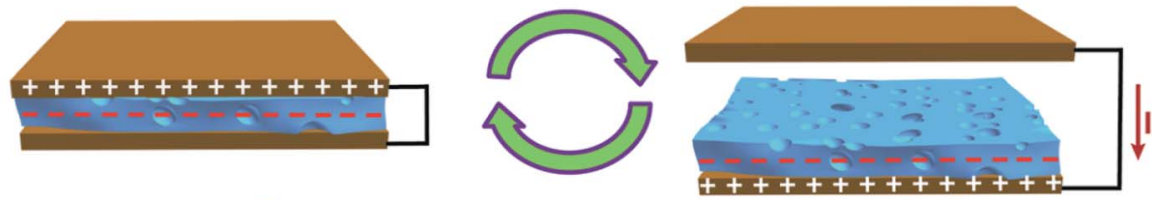

Calcium alginate

Aluminum film

(b)

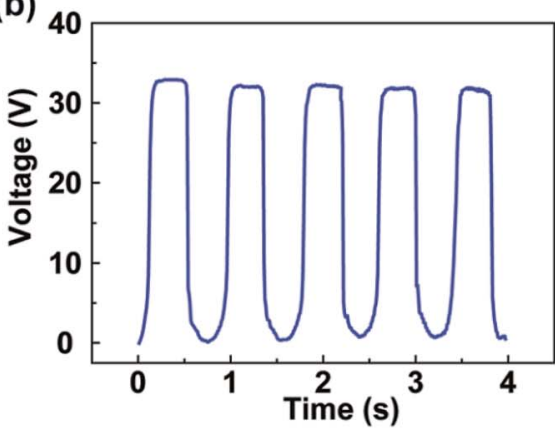

(d)

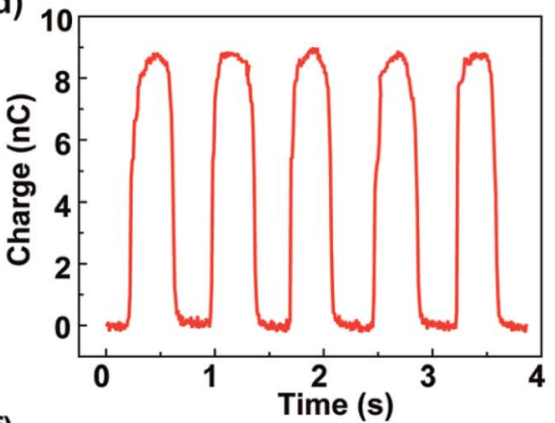

(f)

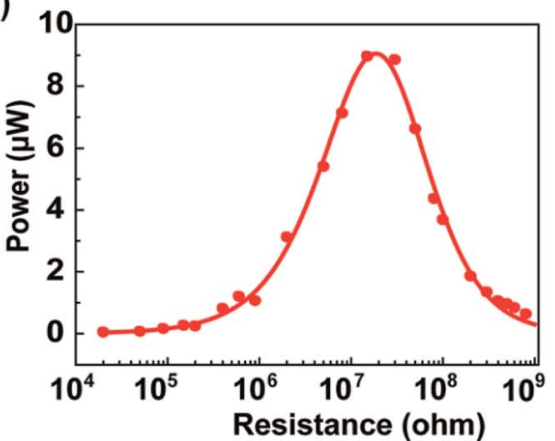

(c)

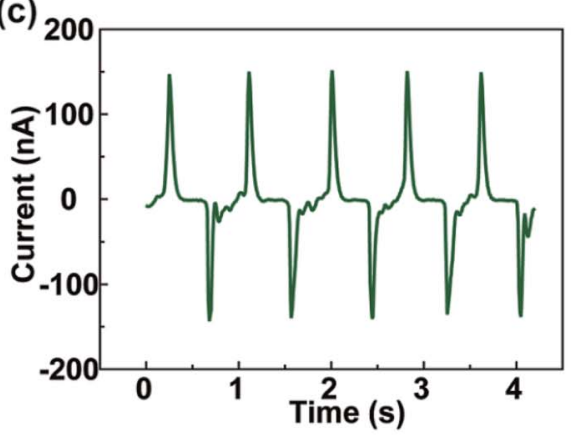

(e)

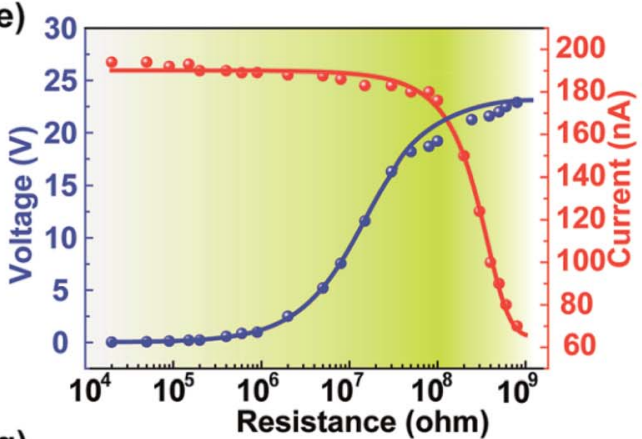

(g)

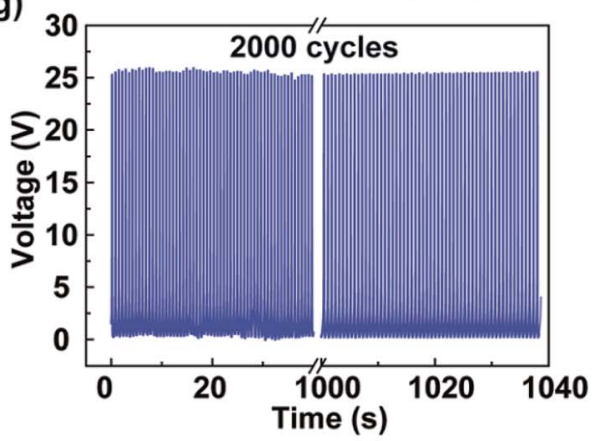

Fig. 2 Energy-harvesting principle and electrical characterization of the degradable TENG. (a) Working principle of the TENG. (b) The opencircuit voltage, (c) short-circuit current, and (d) transferred charge of the fabricated TENG. (e) The relationship between the output voltage/ current and the resistance to an external resistive load. (f) The relationship between the instantaneous power and the resistance to the external resistive load. The maximum instantaneous power is received about $9.5 \mu \mathrm{W}$ when the external load is $20 \mathrm{M} \Omega$. (g) The mechanical durability test of the TENG, where the open-circuit voltage is determined for 2000 cycles. 
(a)
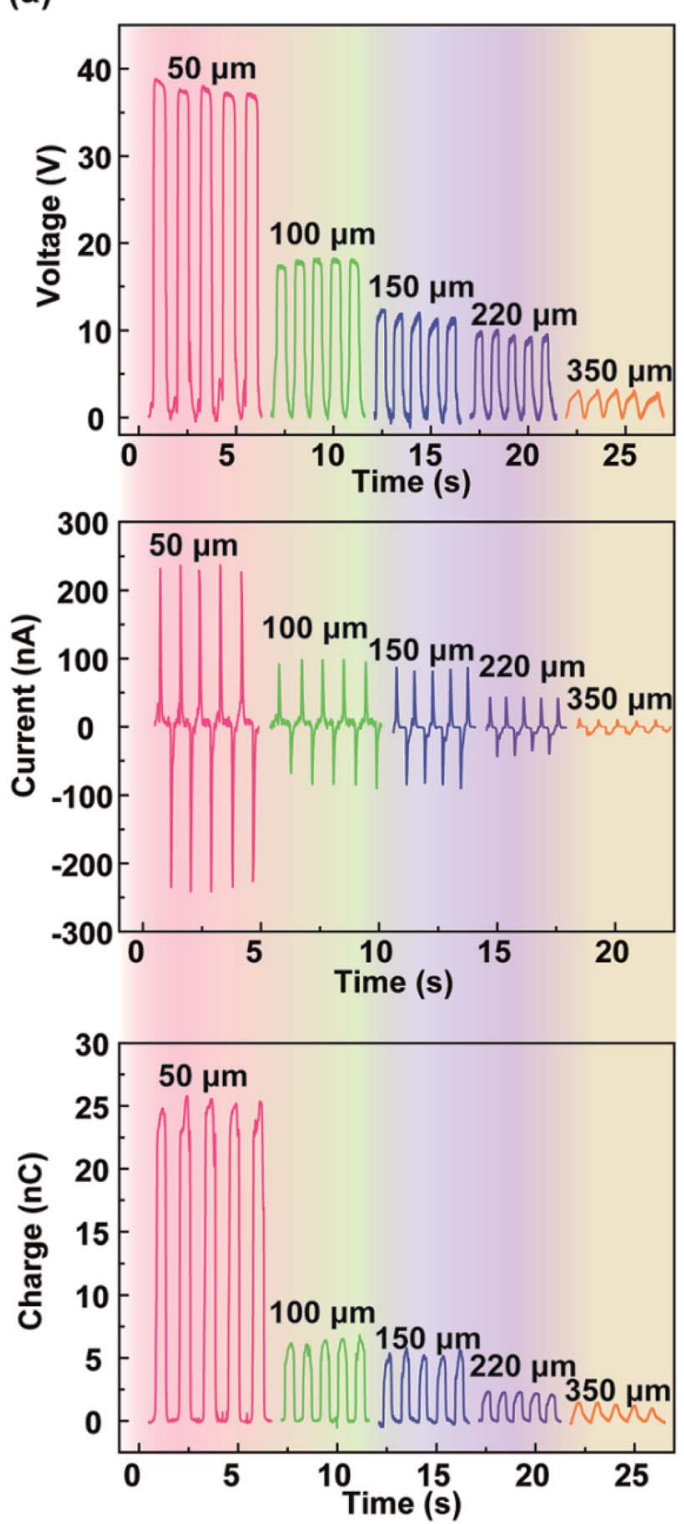

(c)

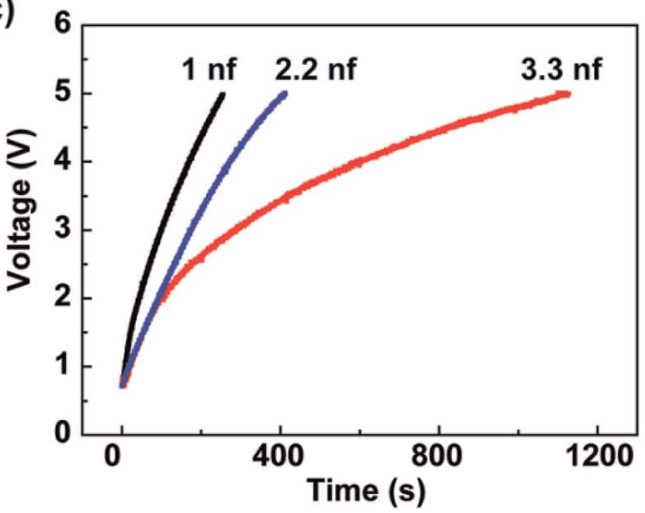

(b)
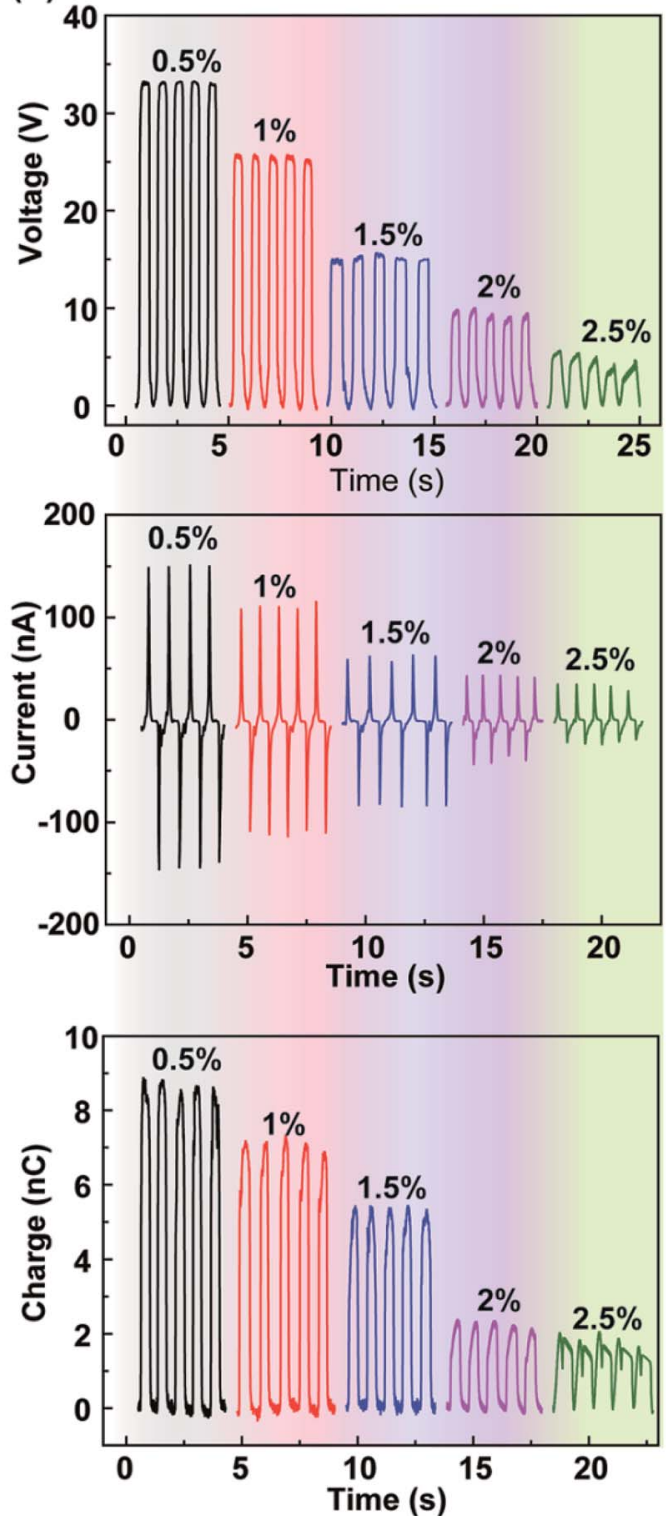

(d)

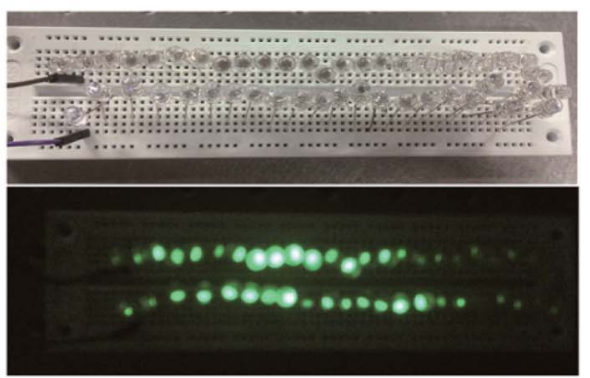

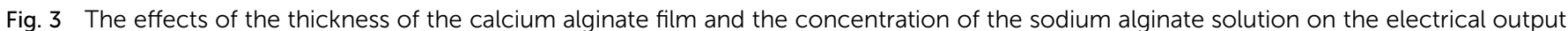

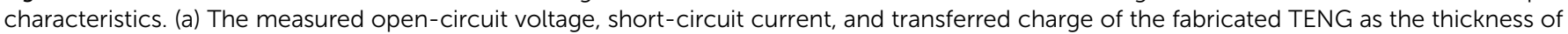

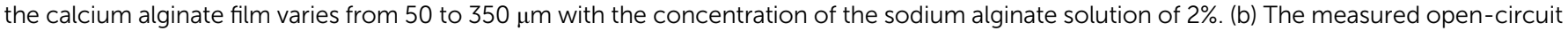

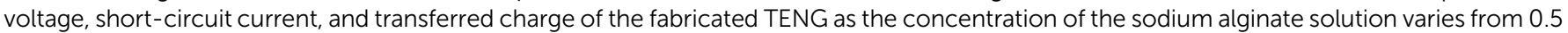

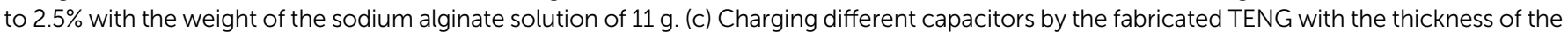

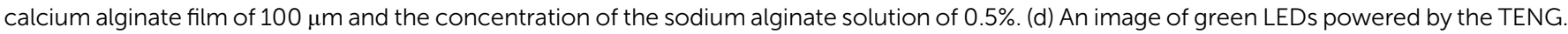


Fig. S2a $\uparrow$ shows the photographic image of the thin calcium alginate film, in which the words on the paper behind the film are clearly visible. Fig. S $2 \mathrm{~b} \dagger$ shows the UV-vis spectrum of the thin calcium alginate film. It can be seen that the film has good optical transparency, which is about $78.6 \%$ in the wavelength range from 300 to $800 \mathrm{~nm}$. Thus, the calcium alginate film has a potential application in the flexible transparent electronics.

The working principle of the alginate film-based triboelectric nanogenerator (TENG) is based on the coupling effects of triboelectrification and electrostatic induction, which is schematically depicted in Fig. 2a. When an external force brings these two friction layers in contact, the electrons are injected from the top $\mathrm{Al}$ electrode into the surface of the calcium alginate film; this leaves the net positive charges on the top Al electrode and the net negative charges on the calcium alginate film. When the two friction layers are separated, there is an electrical potential between these two films that drives the electrons through the external loads. The electrical characteristics of the alginate film-based TENG were investigated. Fig. $2 b-d$ show the open-circuit voltage, short-circuit current, and transferred charge of the fabricated TENG. As can be seen, the open-circuit voltage, short-circuit current, and transferred charge can reach $33 \mathrm{~V}, 150 \mathrm{nA}$, and $9 \mathrm{nC}$, respectively. Fig. 2e shows the resistance dependence of both open-circuit voltage and short-circuit current from $10 \mathrm{k} \Omega$ to $1 \mathrm{G} \Omega$. With an increase in resistance, the open-circuit voltage generally increases and then saturates when the resistance further increases. The short-circuit current shows the opposite trend with an increase in resistance. The relationship between the instantaneous power and the resistance of the external resistive load is also studied. As shown in Fig. 2f, the instantaneous power reaches its maximum of $9.5 \mu \mathrm{W}$ at a resistance of $20 \mathrm{M} \Omega$. Moreover, the mechanical durability of the TENG is measured. As shown in Fig. 2g, the open-circuit voltage remains very stable after 3000 cycles; this demonstrates that the fabricated TENG has an excellent mechanical durability. In addition, we have studied the influence of the surface area on the triboelectric effect. As shown in Fig. S3, $\uparrow$ the open-circuit voltage, short-circuit current, and transferred charge of the fabricated TENG with the surface area from $2 \times 2$ $\mathrm{cm}^{2}$ to $5 \times 5 \mathrm{~cm}^{2}$ are tested. It is observed that the output characteristics of the TENG increase with an increase of the surface area.

The output performances of the fabricated TENG depend on some key design parameters such as the thickness of the calcium alginate film and the concentration of the sodium alginate solution. Fig. 3a shows the open-circuit voltage, shortcircuit current, and transferred charge of the fabricated TENG with various thicknesses of the calcium alginate film of 50, 100, 150,220 , and $350 \mu \mathrm{m}$. It can be found that the open-circuit voltage, short-circuit current, and transferred charge decrease with an increase of the film thickness with the maximum values of $38 \mathrm{~V}, 245 \mathrm{nA}$, and $25 \mathrm{nC}$ at $50 \mu \mathrm{m}$, respectively. The experimental results are in accordance with the theoretical analysis reported in the previous study. ${ }^{34}$ Similarly, Fig. $3 \mathrm{~b}$ shows the measured open-circuit voltage, short-circuit current, and transferred charge of the fabricated TENG as a function of the

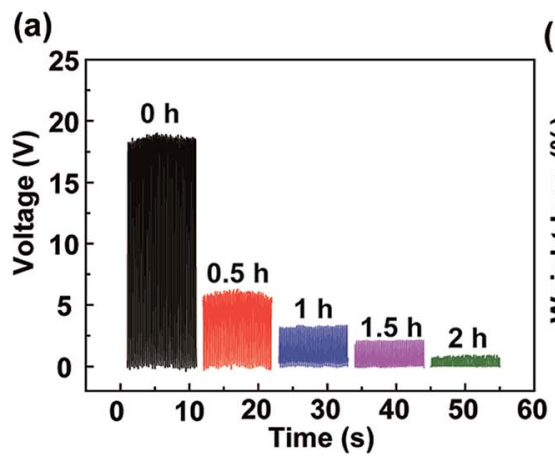

(b)
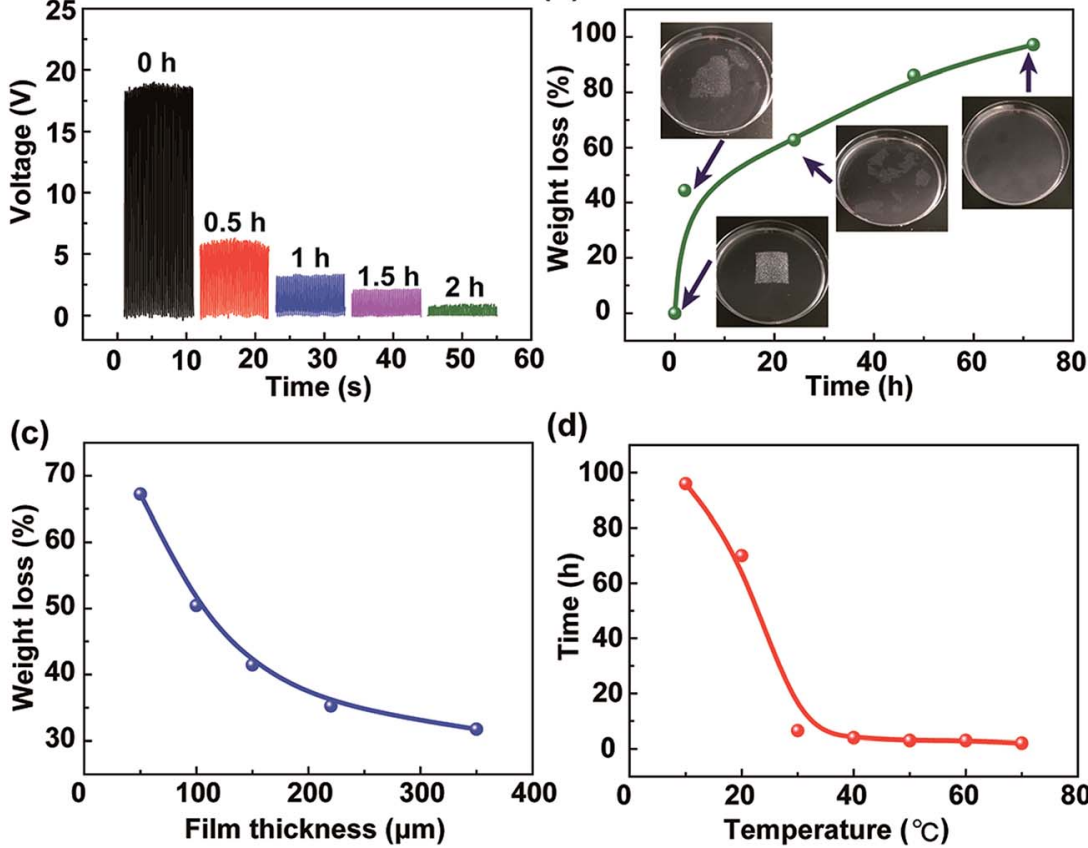

(d)

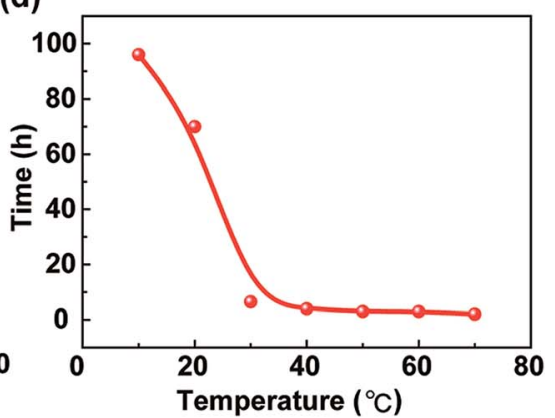

Fig. 4 Degradation process of the calcium alginate film. (a) The output voltage of the fabricated TENG with the degradation time. (b) Weight loss of the calcium alginate film with the thickness of $100 \mu \mathrm{m}$ depended on the degradation time at room temperature. The insets show the images indicating the degradation process of the calcium alginate film. (c) Weight loss of the calcium alginate film depended on the film thickness after $12 \mathrm{~h}$ at room temperature. (d) The complete degradation time of the calcium alginate film with the thickness of $100 \mu \mathrm{m}$ depended on the environment temperature. 
concentration of the sodium alginate solution ranging from $0.5 \%$ to $2.5 \%(\mathrm{w} / \mathrm{w})$. In this experiment, different concentrations of the sodium alginate solution with the same weight of $11 \mathrm{~g}$ were used to fabricate different calcium alginate films. The output characteristics substantially decrease with increasing sodium alginate solution concentrations. The results show that the open-circuit voltage, short-circuit current, and transferred charge reach the maximum values of $34 \mathrm{~V}, 150 \mathrm{nA}$, and $8.8 \mathrm{nC}$, respectively, with the solution concentration of $0.5 \%$. Fig. $\mathrm{S} 4 \dagger$ shows the SEM images of the alginate film with different concentrations. It can be seen that with an increase of the solution concentration, the porosity of the film increases as well. Actually, using the freeze-drying method, water can be adequately removed from the solution, and only the porous film is reserved. Thus, with the increasing concentration of the sodium alginate solution, the film thickness increases, and the output characteristics decrease. Because the capacitance of the film is very important to the performance of the TENG, ${ }^{35,36}$ the capacitances of the film with different concentrations have been studied, as shown in Fig. S5. $\uparrow$ Furthermore, the power harvested by the TENG can be rectified and stored in an energy storage device. As shown in Fig. 3c, three capacitors of 1, 2.2, and $3.3 \mathrm{nF}$ are charged by the fabricated TENG with the thickness of the calcium alginate film of $100 \mu \mathrm{m}$ and the concentration of the sodium alginate solution of $0.5 \%$. They are charged to $5 \mathrm{~V}$ with 253, 411, and 1120 s. As shown in Fig. 3d, the TENG is successfully demonstrated as a power source for simultaneously and continuously lighting up 50 commercial LEDs.

In most cases, the TENG is composed of metal electrodes and a polymer such as PTFE, Kapton, PDMS, and PET, which is difficult to be degraded and liable to release hazardous chemicals. The degradable TENG is composed of $\mathrm{Al}$ electrodes and calcium alginate film, which can be corroded and degraded, respectively. The degradation of the calcium alginate film is systematically studied, as shown in Fig. 4. In this experiment, the calcium alginate film with the thickness of $100 \mu \mathrm{m}$ is immersed in a $3.5 \% \mathrm{NaCl}$ solution, which is used to simulate seawater. Fig. 4a shows that the output voltage of the fabricated TENG dramatically reduces with the increase of the degradation time. When the TENG is immersed in the NaCl solution over $2 \mathrm{~h}$, the calcium alginate film shows serious damage, and the TENG cannot provide the output voltage. Fig. $4 \mathrm{~b}$ shows the (a)

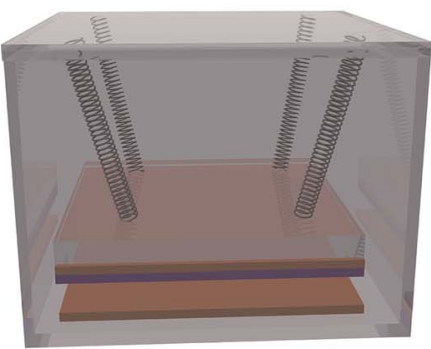

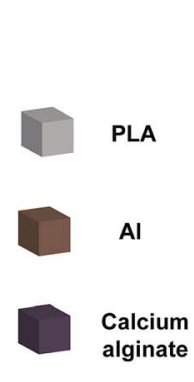

(d)
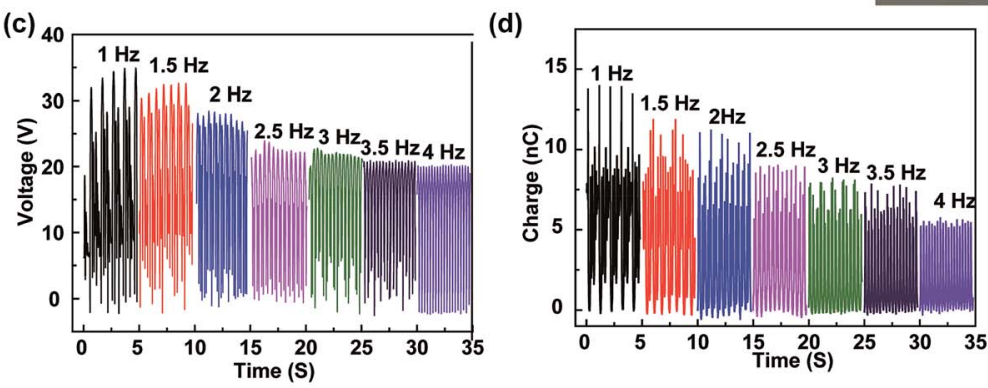

(g)

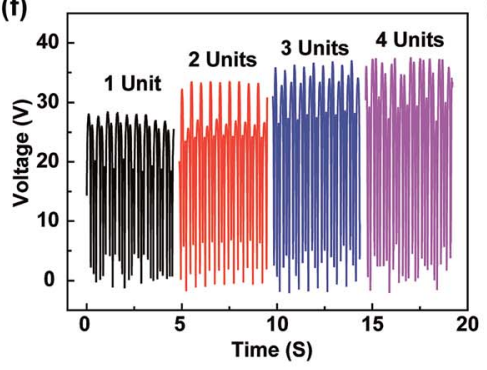

(b)
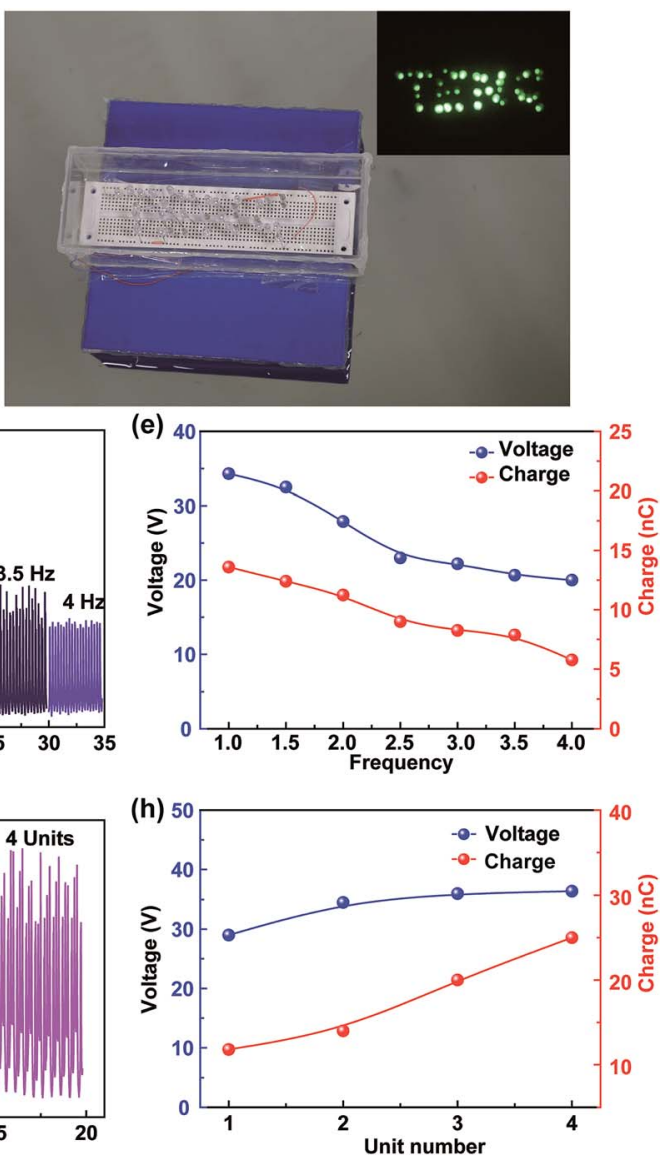

Fig. 5 The structure and electrical output characteristics of the designed TENG for harvesting water wave energy. (a) The device structure of the designed TENG for harvesting water wave energy. (b) The image of the sealed TENGs floating on water and the optical images of LEDs driven by the water wave. (c) The measured open-circuit voltage and (d) the transferred charge of the fabricated TENG at different vibrational frequencies. (e) The relationship between the output characteristics of the designed TENG and the vibrational frequencies. (f) The measured open-circuit voltage and $(\mathrm{g})$ the transferred charge of the fabricated TENG at different units. (h) The relationship between the output characteristics of the designed TENG and the unit number. 
relationship between the weight loss of the calcium alginate film and the degradation time. The insets show the images of the degradation process of the calcium alginate film. It indicates that after $72 \mathrm{~h}$ at room temperature, the calcium alginate film gradually breaks and can be completely degraded with the weight loss approaching $100 \%$. Moreover, the weight loss of the calcium alginate film after $12 \mathrm{~h}$ with different film thicknesses is studied, as shown in Fig. 4c. It needs to be noted that the 50 $\mu \mathrm{m}$ calcium alginate film can be degraded with $67 \%$ weight loss, whereas the $350 \mu \mathrm{m}$ calcium alginate film can only be degraded with $32 \%$ weight loss. Moreover, the relationship between the complete degradation time and the environment temperature is systematically investigated. In this experiment, using the highlow temperature test chamber, the environment temperature can be accurately controlled. The calcium alginate film is immersed in the $\mathrm{NaCl}$ solution at different temperatures until the weight loss approaches $100 \%$. As shown in Fig. 4d, when the temperature is low, the film is difficult to degrade. However, when the temperature is higher than $30{ }^{\circ} \mathrm{C}$, the degradation time is declined sharply. This is because the high temperature will speed up ion exchange and reduce the film structural stability. As abovementioned, the experimental results have indicated that the alginate film-based triboelectric nanogenerator can be recycled and degraded and thus will not cause pollution in the environment.

A kind of sealed degradable triboelectric nanogenerator is designed and fabricated, as shown in Fig. 5a. The TENG is composed of a polylactic acid (PLA) box, springs, and friction surfaces. PLA is a kind of green biodegradable material, and the box is made by the 3D printing technology. The electrodes and springs use the common metal rather than the heavy metals, and the amounts are very small, which will be corroded in the ocean, and the corrosion trace element is necessary for ocean plant growth. ${ }^{37}$ To prevent the instability and improve the output performances of the device, four soft springs are attached between the box and the PLA mass block. For application in wave energy harvesting, four TENG units are integrated into a sealed PLA case, in which the TENG units are connected to a rectifier bridge and then electrically connected in parallel. As shown in Fig. 5b, a sealed device floats on water in a pool, in which the water wave is produced by a wave maker. As shown in Video $\mathbf{S 1}, \dagger$ with fluctuation of water, the scores of LEDs can be lightened up by the wave energy. To study the output characteristics of the TENG, a vibration shaker is used to simulate the fluctuation motion with the operation frequencies ranging from 1 to $4 \mathrm{~Hz}$. The open-circuit voltage and transferred charge of the fabricated TENG at different vibrational frequencies are measured, as shown in Fig. $5 \mathrm{c}$ and d, respectively. Fig. 5e shows the relationship between the output characteristics of the designed TENG and the vibrational frequency. It can be seen that the open-circuit voltage and transferred charge decrease with the increase of the frequency; this is because the friction surfaces cannot fully contact with the increasing vibrational frequency. In particular, the typical wave frequency of the ocean is less than $2 \mathrm{~Hz}^{38,39}$ The measurement results demonstrate that the fabricated TENG has good output characteristics at low-frequency; this is appropriate for harvesting the wave energy. Furthermore, as shown in Fig. $5 \mathrm{f}$ and $g$, the effect of the unit number on the output performance of the fabricated TENGs is studied. Fig. $5 \mathrm{~h}$ shows the relationship between the output characteristics of the designed TENG and the unit number. As can be seen, the open-circuit voltage increases with the increasing unit number. The transferred charge of one unit is about $10 \mathrm{nC}$, and it is greatly increased to $25 \mathrm{nC}$ at 4 units. The experimental results indicate that the fabricated TENG can be degradable and ocean environmentfriendly and has a great application in blue energy harvesting.

\section{Conclusions}

In summary, a degradable triboelectric generator based on an alginate film has been proposed. Calcium alginate is extracted from the ocean plants and fabricated to a film using the simple freeze-drying method. The fabricated calcium alginate film has a porous structure, and its surface is rough, which is beneficial for triboelectric power generation. The fabricated TENG has good output performance with a maximum voltage, current, and power of $33 \mathrm{~V}, 150 \mathrm{nA}$, and $9.5 \mu \mathrm{W}$, respectively. The fabricated TENG has an excellent performance in the stability test, and the output voltages are nearly invariable after 2000 cycles. Furthermore, the effects of the thickness of the calcium alginate film and the concentration of the sodium alginate solution on the electrical output characteristics as well as the thickness and temperature on the degradability are investigated. In addition, the TENG is designed for harvesting water wave energy with different frequencies and unit numbers. Using degradable materials based on the ocean plants in the TENG, a new insight is achieved to develop eco-friendly power sources and expand the application range in blue energy.

\section{Experimental}

The output voltage signals, charge transfers, and current signals of the device were acquired via the Keithley 6514 System Electrometers. The SEM images of the calcium alginate film were obtained by SU 8020 . The FTIR spectra of the calcium alginate and sodium alginate were obtained by VERTEX 80V.

\section{Conflicts of interest}

There are no conflicts to declare.

\section{Acknowledgements}

The authors acknowledge the support provided by the National Key Research and Development Program of China (2016YFA0202704), the National Natural Science Foundation of China (No. 51475099), the Beijing Natural Science Foundation (No. 4163077), the Beijing Nova Program (No. Z171100001117054), the Youth Innovation Promotion Association, CAS, the Beijing Municipal Science \& Technology Commission (Z171100000317001), and the "thousands talents" program for the pioneer researcher and his innovation team, China. 


\section{Notes and references}

1 B. Z. Tian, X. L. Zheng, T. J. Kempa, Y. Fang, N. F. Yu, G. H. Yu, J. L. Huang and C. M. Lieber, Nature, 2007, 449, 885-889.

2 J. Scruggs and P. Jacob, Science, 2009, 323, 1176-1178.

3 M. Gross, Sci. Commun., 2013, 35, 810-818.

4 S. Xu, Y. Qin, C. Xu, Y. G. Wei, R. S. Yang and Z. L. Wang, Nat. Nanotechnol., 2010, 5, 366-373.

5 Z. L. Wang and W. Z. Wu, Angew. Chem., Int. Ed., 2012, 51, 11700-11721.

6 Z. L. Wang and J. H. Song, Science, 2006, 312, 242-246.

7 F. R. Fan, Z. Q. Tian and Z. L. Wang, Nano Energy, 2012, 1, 328-334.

8 Z. L. Wang, Mater. Today, 2017, 20, 74-82.

9 C. B. Han, C. Zhang, W. Tang, X. H. Li and Z. L. Wang, Nano Res., 2015, 8, 722-730.

10 T. Zhou, L. M. Zhang, F. Xue, W. Tang, C. Zhang and Z. L. Wang, Nano Res., 2016, 9, 1442-1451.

11 C. Zhang, W. Tang, C. B. Han, F. R. Fan and Z. L. Wang, Adv. Mater., 2014, 26, 3580-3591.

12 W. Tang, C. Zhang, C. B. Han and Z. L. Wang, Adv. Funct. Mater., 2014, 24, 6684-6690.

13 H. D. Hou, Q. K. Xu, Y. K. Pang, L. Li, J. L. Wang, C. Zhang and C. W. Sun, Adv. Sci., 2017, 1700072.

14 Y. K. Pang, X. H. Li, M. X. Chen, C. B. Han, C. Zhang and Z. L. Wang, ACS Appl. Mater. Interfaces, 2015, 7, 19076-19082.

15 L. M. Zhang, F. Xue, W. M. Du, C. B. Han, C. Zhang and Z. L. Wang, Nano Res., 2014, 7, 1215-1223.

16 J. J. Luo, W. Tang, R. F. Fan, C. Liu, Y. K. Pang, G. Cao and Z. L. Wang, ACS Nano, 2016, 10, 8078-8086.

17 C. B. Han, T. Jiang, C. Zhang, X. H. Li, C. Y. Zhang, X. Cao and Z. L. Wang, ACS Nano, 2015, 9, 12552-12561.

18 X. Pu, M. Liu, L. Li, C. Zhang, Y. Pang, C. Jiang, L. Shao, W. Hu and Z. L. Wang, Adv. Sci., 2016, 3, 1500255.

19 X. X. Pu, H. Y. Guo, J. Chen, X. Wang, Y. Xi, C. G. Hu and Z. L. Wang, Sci. Adv., 2017, 3, e1700694.

20 C. Zhang, W. Tang, L. M. Zhang, C. B. Han and Z. L. Wang, ACS Nano, 2014, 8, 8702-8709.
21 C. Zhang, L. M. Zhang, W. Tang, C. B. Han and Z. L. Wang, Adv. Mater., 2015, 27, 3533-3540.

22 C. Zhang and Z. L. Wang, Nano Today, 2016, 11, 521-536.

23 Y. K. Pang, J. Li, T. Zhou, Z. W. Yang, J. J. Luo, L. M. Zhang, G. F. Dong, C. Zhang and Z. L. Wang, Nano Energy, 2017, 31, 533-540.

24 L. Xu, Y. K. Pang, C. Zhang, T. Jiang, X. Y. Chen, J. J. Luo, W. Tang, X. Cao and Z. L. Wang, Nano Energy, 2017, 31, 351-358.

25 L. M. Zhang, C. B. Han, T. Jiang, T. Zhou, X. H. Li, C. Zhang and Z. L. Wang, Nano Energy, 2016, 22, 87-94.

26 T. Jiang, L. M. Zhang, X. Y. Chen, C. B. Han, W. Tang, C. Zhang, L. Xu and Z. L. Wang, ACS Nano, 2015, 9, 1256212572.

27 M. M. Osman, Mater. Chem. Phys., 2001, 71, 12-16.

28 E. M. Sheirf, Int. J. Electrochem. Sci., 2011, 6, 1479-1492.

29 K. Y. Lee and D. J. Mooney, Prog. Polym. Sci., 2012, 37, 106126.

30 W. H. Tan and S. Takeuchi, Adv. Mater., 2007, 19, 2696-2701.

31 S. N. Pawar and K. J. Edgar, Biomaterials, 2012, 33, 32793305.

32 J. S. Yang, Y. J. Xie and W. He, Carbohydr. Polym., 2011, 84, 33-39.

33 Y. Q. Li, H. Zhang, M. Z. Fan, P. T. Zheng, J. D. Zhuang and L. H. Chen, Sci. Rep., 2017, 7, 46379.

34 S. M. Niu, S. H. Wang, L. Lin, Y. Liu, Y. S. Zhou, Y. F. Hu and Z. L. Wang, Energy Environ. Sci., 2013, 6, 3576-3583.

35 X. He, H. Guo, X. Yue, J. Guo, Y. Xi and G. Hu, Nanoscale, 2017, 5, 1896.

36 J. Chen, H. G., X. He, G. Liu, Y. Xi, H. Shi and C. Hu, ACS Appl. Mater. Interfaces, 2016, 8, 736-744.

37 P. W. Boyd, T. Jickells, C. S. Law, S. Blain, E. A. Boyle, K. O. Buesseler, K. H. Coale, J. J. Cullen, H. J. W. de Baar, M. Follows, M. Harvey, C. Lancelot, M. Levasseur, N. P. J. Owens, R. Pollard, R. B. Rivkin, J. Sarmiento, V. Schoemann, V. Smetacek, S. Takeda, A. Tsuda, S. Turner and A. J. Watson, Science, 2007, 315, 612-617.

38 Z. L. Wang, T. Jiang and L. Xu, Nano Energy, 2017, 39, 9-23. 39 M. F. Abu Riduan and C. Gwiy-Sang, J. Semicond., 2012, 33, 074001. 